\title{
Recent advances in characterisation of subsonic axisymmetric nozzles
}

\author{
Václav Tesař ${ }^{1, a}$ \\ ${ }^{1}$ Institute of Thermomechanics v.v.i., Czech Academy of Sciences, Dolejškova 5, 18200 Praha-Kobylisy, Czech Republic
}

\begin{abstract}
Nozzles are devices generating jets. They are widely used in fluidics and also in active control of flows past bodies. Being practically always a component of larger system, design and optimisation of the system needs characterisation of nozzle properties by an invariant quantity. Perhaps surprisingly, no suitable invariant has been so far introduced. This article surveys approaches to characterisation quantities and presents several examples of their typical use in systems such as parallel operation of two nozzles, matching a nozzle to its fluid supply source, apparent resistance increase in flows with pulsation, and the secondary invariants of a family of quasi-similar nozzles.
\end{abstract}

\section{Introduction}

Nozzles generate jets by allowing fluid to leave closed cavities and issue into a more or less open space. Some uses of nozzles are long established, in fact since prehistoric times, shown by example Fig. 1. Quite old are also various uses in heating and drying by impinging jets [2]. Recently rapidly increasing in importance are nozzles for jet control of flow past bodies (airplane wings or turbine blades [7]), where the jets replace earlier mechanical components (flaps, flow spoilers, etc.) inserted into the flow. Substantially dependent on nozzles is also fluidics, the technique of generation and control of fluid flows. It recently became widely used especially in the small scale microfluidics [3] and also - with large potential in energy engineering - in fuel producing bioreactors [8] or the new approaches to combustion [6].

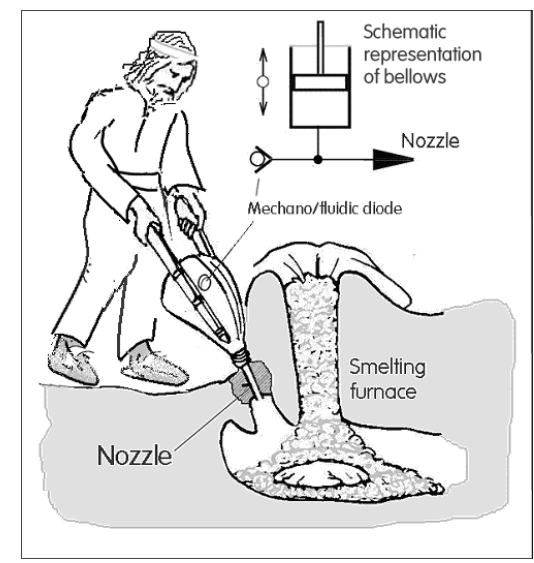

Fig. 1. An example of earliest use of nozzles. Since the Early Bronze Age ( 4000 B.C.) nozzles were applied to increasing the temperature in producing metals from ore [4]. Air is here seen being blown into the smelting furnace by bellows through a ceramic nozzle. Reminders of such nozzles are found in large quantities in archaeological excavations [5].

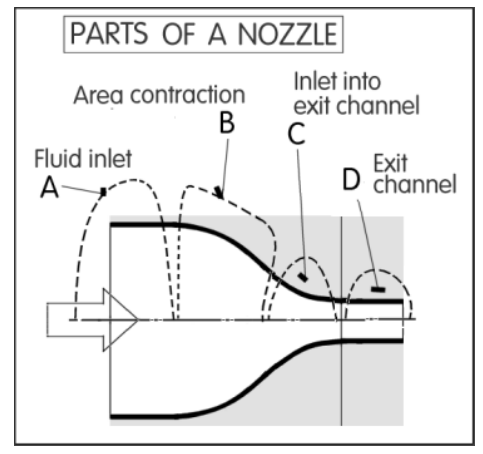

Fig. 2. Component parts of typical low-loss (i.e. smoothly contoured) axisymmetric nozzle. Fluid is accelerated in the area contraction B to counter the slowing down of the generated jet by viscous and vortex momentum transport to surroundings.

\section{Nozzles}

Nozzles - even if limited in this article to only subsonic and axisymmetric versions - exist in vast numbers of various shapes. In contrast to diffusers (which perform the opposite conversion of deceleration, Fig. 3), design of which for good performance may be difficult, nozzles pose less problems. Almost any nozzle geometry is likely to perform acceptably well. This is reflected in literature, vast one about diffusers and much less extensive about nozzles. In fact the nozzle literature does not very much address the question of pressure difference across nozzles discussed in this paper. Instead, it mostly concentrates on ensuring a particular shape of velocity profile at the nozzle exit. Especially wind tunnel nozzles are required to generate a special, rectangular profile. To exhibit low loss, nozzles require rounding all internal edges, as shown in the shape example in Fig. 2. The rounding can be made relatively easily if the nozzle is large (as in the wind tunnel case). At small scales the rounding may be a problem since it may be difficult to get with the machine

a Corresponding author: tesar@it.cas.cz 


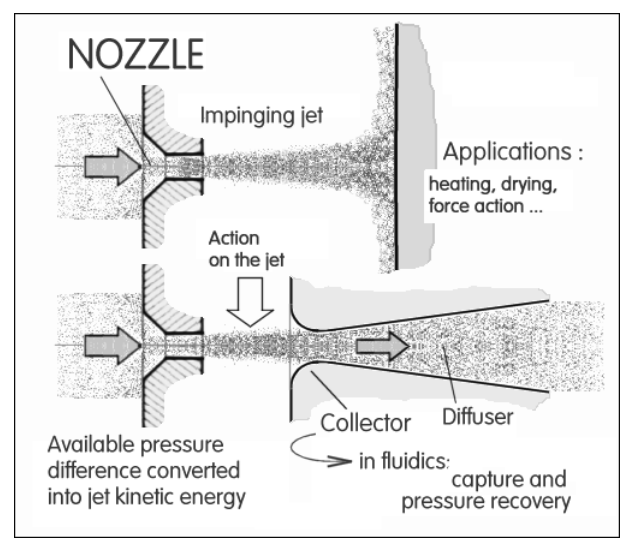

Fig. 3. There are applications with complicating effects caused by a device exposed to the jet flow. Fortunately, the impingement (top) and jet-capturing-collector flows (bottom) do not contribute significantly to pressure difference across the nozzle - the problem discussed here.

tool to the internal surfaces. Making small nozzles thus may require a special manufacturing process (e.g., the "3D printing"), which tend to be slow and expensive. As a result, unless there is requirement of very low loss, small size nozzles are quite often made with manufacturing economy as the chief factor. Nozzles are thus not infrequently made with the sharp edges as left by drilling - or, as a compromise, with just the entrance edge removed by chamfering [11] as seen in Fig. 4 .

Present paper discusses axisymmetric nozzles with round exit channel of diameter $d$ (Fig. 5). Typical feature of most nozzles is the decrease of area cross section in the flow direction in the locations $B$ and $C$ in Fig. 2. The reason is to counter the gradual momentum loss of generated jet especially if - as is often the case - the jet is required to reach with a reasonable velocity to a certain axial distance from the nozzle exit. The momentum loss is particularly remarkable if the jet is of submerged type, i.e. if the jet fluid is gas issuing into a gas-filled space or a liquid jet is issuing into space filled with liquid).

The problem caused by the sharp edges of the convex type "a" in Fig. 4, is they give rise to separation of the flow from the walls downstream from the edge - forming there an annular vortex ring. This vortex tends to

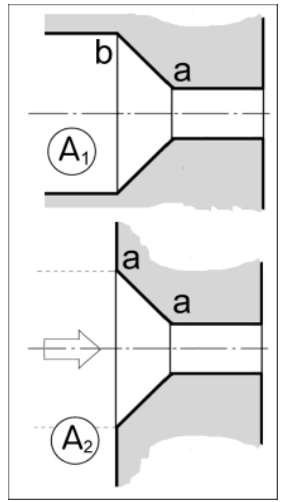

Fig. 4. Fluid is supplied into a nozzle using one of these two inlet configurations: either by the pipe entry $A_{1}$ or from the halfspace $A_{2}$. The effect of these inlets on nozzle pressure drop is fortunately insignificant. More important are vortices generated at the convex corners "a" and concave "b". be shed, usually in an irregular manner. The result is unwelcome pulsation in the generated jet flow. Also, the vortex shedding extracts from the main flow the energy tneeded for generating a new ring that replaces the shed one. Similar re-circulating vortex rings are also formed in the concave corner "b" shown in Fig. 4. This is energetically less demanding (velocity of motion inside the corner "b" is lower), but the unpleasant effect of vortex shedding may be found even there.

An important feature of good nozzles is their exit channel (the part $D$ in Fig. 2). Its task is to stabilise the generated jet and ensure its proper flow direction. In an attempt to decrease the friction losses some nozzle designers disregard the need of this channel and make it short or absent. It is not a good idea. To be effective, the exit channel should not be shorter than $\sim 2 \mathrm{~d}$. A shorter exit channel or missing immediately downstream from the contraction generates at the exit a phenomenon known under the historic term "vena contracta", causing significant energetic losses.

Only in a few cases (room heating, ventilation) the jets are left to dissipate their kinetic energy completely in the downstream space. Much more often they are used to perform there various actions like impingement (top of Fig. 3), or are captured into a collector (bottom of the picture). A literature on these phenomena exists, like [2] or [3]. From the point of view of the present article these phenomena are generally of little relevance. Perhaps more important may be the pressure decrease downstream from the nozzle exit due to the jet-pumping effect (entrainment of the outer fluid and dragging it along with the jet). Also this effect on nozzle pressure drop is relatively negligible unless the objects on which the jet acts are at very short distance from the nozzle exit.

\section{Characterisation of nozzles in their role of circuit components}

Prior to development of no-moving-part fluidics, the hydraulic and pneumatic systems almost always operated with some mechanical movable components. From the systems point of view these arrangements were usually quite simple. Their design could be thus handled by classical fluid mechanics, evaluating local velocity and local pressure - and then integrating these values to compute the acting forces. It was fluidics [3] that came with circuit solutions ideas that follow the well developed analogous theory of circuits in electric engineering. Unfortunately, in this approach fluidics encountered several significant problems. Behaviour of the components from which electric circuits are built is in steady states fully described by resistance $R$. This characterises the dependence between two parameters of state, the current $\mathrm{I}[\mathrm{A}]$ and voltage difference $\Delta \mathrm{U}[\mathrm{V}]$, mutually related by the simple linear Ohm's law,

$$
\Delta \mathrm{U}=\mathrm{R} \text { I }
$$

In the case of fluidic devices - and nozzles in particular - the situation is very much different.

(a) While the electric state variables are directly measurable by instruments, in fluidics the values measured by manometer and flowmeter are insufficient. 
They serve just as inputs into a more complex computation procedure [21]. Pressure difference $\Delta P[P a]$ measured by manometers may be considered an analogy to $\Delta \mathrm{U}$ in eq. (1) - while the role of the through variable, corresponding to the electric current, may be assumed by the volume flow rate $\dot{V}\left[\mathrm{~m}^{3} / \mathrm{s}\right]$ measured by flowmeters. In an electric system, the solution then progresses by input of the measured quantities into the Kirchoff laws, basically conservation relations for circuit solutions. Unfortunately, there are no conservation laws for the quantities $\Delta \mathrm{P}$ and $\dot{\mathrm{V}}$. Obviously, in a real fluid of non-negligible compressibility its volume is not constant and increases along the pressure decrease. Thus $\forall$ typically grows in the flow direction. The problems with $\Delta \mathrm{P}$ as opposed to decrease of $\Delta \mathrm{U}$ in the direction of electric current is particularly apparent in a diffuser the pressure in which may actually increase in the direction of fluid flow. Yet $\Delta \mathrm{P}$ and $\dot{V}$ were used traditionally and it took considerable time and effort to break this tradition to accept the introduction of the not directly measurable conservation quantities, the specific energy difference $\Delta \mathbf{e}\left[\mathrm{m}^{2} / \mathrm{s}^{2}\right]$ together with the mass flow rate $\mathrm{M}[\mathrm{kg} / \mathrm{s}]$.

b) In contrast to the simple across variable $\Delta U$ in eq. (1), the specific energy difference $\Delta \mathbf{e}$, which plays in fluidics the analogous role, is a multi-component vari-able. The drop in specific energy across a nozzle (or another fluidic device) is due to a part of it being spent on driving the fluid through. It is a sum of differences is several (usually three) components - as discussed in eq. (1) in [11]:

$$
\Delta e=\left(e_{k}+e_{P}+e_{p o s}\right) x-\left(e_{k}+e_{P}+e_{p o s}\right) y
$$

- between an inlet $X$ and exit $Y$. In eq. (2) $e_{k}$ is specific kinetic energy of fluid while specific sum of pressure and thermal energy is ep. If the fluid is not gas, it is necessary to consider also the difference in specific vertical position energy of the liquid), which is $e_{\text {pos. }}$.

c) In contrast to the Ohm's law eq. (1), fluidic devices mostly behave in nonlinear manner, complicating the solution mathematics.

Since the dissipated energy in eq.(2) is mostly the kinetic component $\Delta \mathrm{e}_{\mathrm{k}}$ (organised flow motion is converted into the chaotic energy of thermal motions) and this is proportional to the square of the velocity, eqs. (3, $4,5)$ in [11], the equation of characteristic for most nozzles is near to quadratic parabola.

$$
\Delta \mathrm{e} \sim \dot{\mathrm{M}}^{2}
$$

An important tool for solutions of problems of fluid mechanics in general is the dimensional analysis and the associated Buckingham's $\pi$ theorem. It simplifies solved problems by reducing the number of variables (by conversion of primary simplexes into complex quantities). A governing equation that involves $n$ simple variables can be re-written as an equation of $n-m$ dimensionless parameters, where $m$ is the number of independent dimensions. This analysis may even suggest the characters of the initially unknown governing equation. Among the dimensionless complex parameters, of essential importance for nozzles is Reynolds number

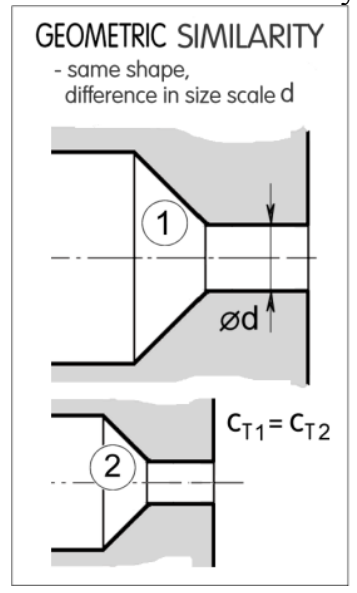

Fig. 5. Two nozzles are fully similar if they differ only in magnitude of the exit diameter $d$. Such full similarity has been always considered a necessary condition for application of the dimensional analysis.

$\operatorname{Re}$ of the nozzle exit flow, eq. (1) in [12].

$$
\operatorname{Re}=\frac{W d}{\nu}
$$

Apart from the size scale expressed by the diameter $d$ and the scale of velocities of motion, expressed by the nozzle exit velocity $w[\mathrm{~m} / \mathrm{s}], R e$ involves also the variable that expresses the properties of the fluid, viscosity $v\left[\mathrm{~m}^{2} / \mathrm{s}\right]$ characterising the magnitude of internal friction - energy dissipation in the fluid

It has been always considered without questions that the basic condition necessary for the similarity solutions is the geometric similarity, as shown in Fig. 5. It means the devices for which the solution is valid must be of the same geometry and differ only in the size scale.

Dynamics of flowfields - the problem of momentum transport in a flow with dependence on more than a single spatial co-ordinate - is described by partial differential equations. Very important consequence of what the similarity approach can do is reducing the number of variables. This is essential simplification mathematics: the partial differential equation with two or even three independent variables is converted into the much easier to solve ordinary differential equation - with derivatives only by the single similarity-transformed independent dimensionless distance. This similarity-based simplification is described, e.g., in [19]. Later, in [14], it was attempted to apply this similarity-transform approach to compute the flowfield of synthetic jets, generated by alternating inflow and outflow from a nozzle. In this case, however, the complicated conditions made the same full similarity solution not possible. Nevertheless, the situation was not completely hopeless. What was possible, is applying the concept of "quasi-similarity". It means the transformation of variables and of the governing partial differential equation was not made at once to be valid over the whole investigated flowfield. Instead, the key parameter in the similarity transformation was gradually varied as the solution progressed along the jet axis.

There is a fundamental principle of physical sciences in claiming that solutions of physical problems in general 


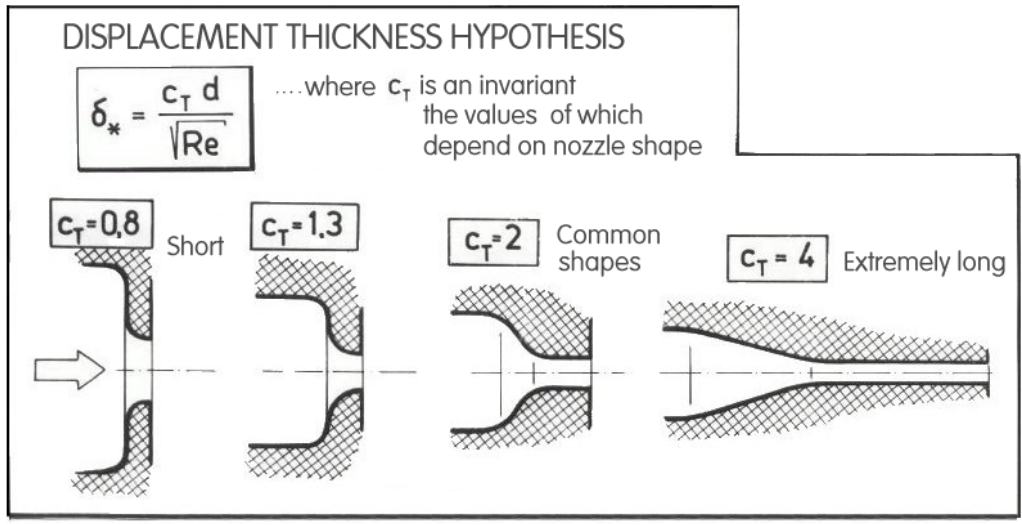

Fig. 6. Characterisation invariant $\mathrm{C}_{\mathrm{T}}$ of nozzles was introduced initially in $[1,9]$ for nozzles with smooth, rounded internal shape but it could be later extended to characterise the internal geometry of nozzles in general. Increasing ст value means a longer friction path of the internal flow, the consequence being a thicker boundary layer in the nozzle exit plane.

are actually identification of problem invariants. In the present case of nozzles, the characterisation invariant was found by this author to be the parameter $c_{T}$, introduced already in 1985 [1] to specify the properties of nozzle geometry. Typical meaning of this characterisation quantity is shown in the example of nozzles of various axial length shown in Fig. 6. Introduction of the parameter $\mathrm{C}_{\mathrm{T}}$ was based in [1] on two hypotheses. Both were valid for smoothly shaped nozzles (no sharp internal edges). An easier to follow explanation of the approach, than [1] is available the later paper [9].

The idea of the invariant characterising a nozzle was later extended to be applicable also to nozzles with the sharp internal edges [10]. This is useful because of the importance of such nozzles in the practical applications requiring inexpensive, simply made nozzles. Analysis of the two ideas [9] and [10] later found that the approach in [10] is actually convertible to $\mathrm{CT}_{\mathrm{T}}$ in [9].

The basic idea of this characterisation is the fact that parameter $\mathrm{C}_{\mathrm{T}}$ fully specifies nozzle behaviour as presented by the characteristic in Fig. 8. In other words, two nozzles with the same magnitude of the shape para- meter Cт exhibit the same specific energy loss $\Delta \mathbf{e}$ at any given flow rate $\dot{M}$ - even though they may be geometrically very much different. This means here is not valid the standard definition of similarity according to which similar nozzles differ only in their absolute size expressed by their exit diameter $d$.

Interestingly, this new idea of similarity may be extended also to quasi-similarity: geometric variability of the numerical value of a shape parameter. In [12] it was applied to a family of nozzles, geometrically otherwise similar apart from having different exit channel lengths, as presented in Fig. 7.

Before advancing to presenting examples of prac-tical use of the nozzle characterisation, it may be useful to discuss the simpler approach to the characterisation problem. It may be seen as member of the following list:

0-th order characterisation: neglects frictional losses inside the nozzle.

$1^{\text {st }}$ order characterisation: takes friction loss into account but considers it constant, not Re-dependent.

$2^{\text {nd }}$ order characterisation: full description of be-haviour in the most important range of Reynolds numbers.

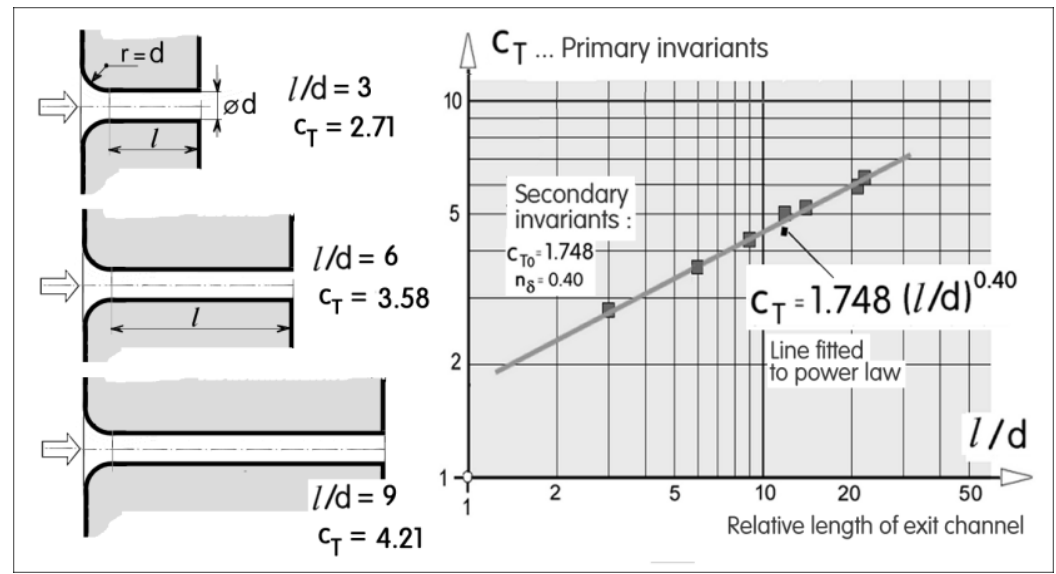

Fig. 7. An example of nozzle family with geometric quasi-similarity of having different exit-channel lengths which makes the standard $\pi$ theorem not applicable. Since in the pressure-difference analysis the interest is only in a single quantity of each nozzle its characterisation invariant $\mathrm{C}_{\mathrm{T}}-$ it became possible to introduce [12] the idea of the secondary invariants, $\mathbf{C}_{\mathrm{T} 0}$ and $\mathbf{n}_{\delta}$. 


\section{Fist-order dissipance $\mathbf{Q}$ \\ characterisation \\ by}

Obvious idea considered useful at the time when were designed and tested first fluidic circuits was to follow the analogy with the circuits theory in electrical engineering. In the steady-state regimes, behaviour is there described by the value of $R$, the multiplicative parameter in the Ohm's law, eq. (1). As already mentioned above, in the fluidic devices the analogy of eq. (1) is nonlinear, nearest perhaps to quadratic dependence.

$$
\Delta \mathrm{e}=\mathrm{Q} \mathrm{M}^{2}
$$

- this is eq. (3) with introduced multiplication factor Q $\left[\mathrm{m}^{2} / \mathrm{kg}^{2}\right]$ known as quadratic dissipance. In many practical situations the deviations of real behaviour from eq. (5) may be small and negligible. Then the numerical value of dissipance $\mathbf{Q}$ may be considered constant — and may be then considered as characterising the first-order behaviour of a nozzle, Fig. 8 .

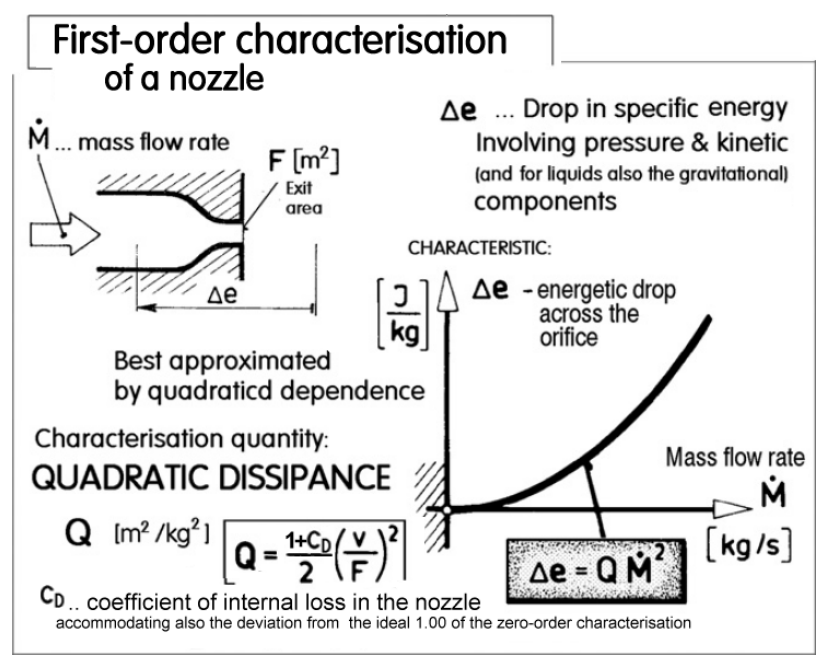

Fig. 8. Nozzles may be approximately characterised by the single parameter $\mathrm{Q}$ in the simple quadratic dependence eq. (5) between the two state parameters. Strictly speaking Q is not an invariant - but deviations are small and handling it as if it were simplifies solution of many typical problems.

\section{Practical uses of characterisation}

In experimental investigation of a nozzle the mass flow rate at the right-hand side of eq. (5) is usually obtained by readings of a flowmeter - while in a numerical flowfield computation its quantity is input by simple summation of inlet velocity boundary condition over the inlet area. The specific energy drop $\Delta \mathrm{e}$ across the nozzle on the lefthand side of eq. (5) may be an object of complicated computations $[3,21]$ if the conditions make it necessary to take into account compressibility of the fluid. If, however, the compres- sibility can be neglected, then $\Delta \mathrm{e}$ is simply proportional to pressure difference $\Delta \mathbf{P}$ measured by a manometer

$$
\Delta \mathrm{e}=\mathrm{V} \Delta \mathrm{P}
$$

- where the proportionality constant $v\left[\mathrm{~m}^{3} / \mathrm{kg}\right]$, is the specific volume of the fluid. For flows of a gas this quantity $\mathrm{v}$ is computed from temperature, pressure, and gas constant $r$ :

$$
V=\frac{r T}{P}
$$

The value of the dissipance $\mathbf{Q}$ is then evaluated as

$$
Q=\left(1+c_{D}\right) \frac{8 v^{2}}{\pi^{2} d^{4}}
$$

-where $\mathbf{C}_{\mathbf{D}}$ is the loss (or drag) coefficient of the internal flow in the nozzle

$$
E u=1+C_{D} \begin{aligned}
& \text { Energy dissipation } \\
& \text { inside nozzle }
\end{aligned}
$$

while Eu is the loss Euler number, mostly involving the dissipation of the kinetic energy of the jet. In the zeroethorder approach, useful for initial assessments of nozzle behaviour, $\mathbf{C}_{\mathbf{D}}$ is neglected and only the jet dissipation is considered

$$
\mathrm{Eu}=1
$$

Second-order characterisation also operates with Euler number. Important property of this approach, however, is variation of Eu with Reynolds number $[9,10]$

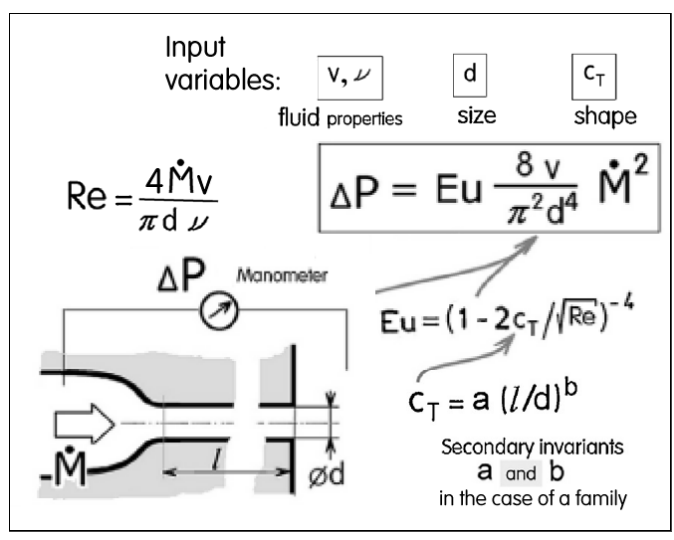

Fig. 9. Schematic representation of the steps in computation of pressure drop across a nozzle from known Ст (or known secondary invariants, Fig. 7). It is assumed that Reynolds number of the nozzle exit flow is $\operatorname{Re}>2000$ (otherwise, in the creeping flow regime, it would be necessary to apply higher characterisation approach [12] ).

After identification of the numerical value of the invariant coefficient $\mathrm{CT}_{\mathrm{T}}$, the $\mathrm{Eu}$ is calculated using the second-order universal characteristic

$$
E U=\frac{\operatorname{Re}^{2}}{C_{T}^{4}} /\left(\frac{\sqrt{R e}}{C_{T}}-2\right)^{4}
$$

Validity is demonstrated using experimental data for two extreme nozzle cases in Fig. 10. On one hand is there he case of extremely long exit channel nozzle $l=13.9 \mathrm{~d}$, (nozzles so long are not used in standard engineering practice) for which was evaluated $\mathrm{C}_{\top}=4.771$. The other case in Fig. 10 is the extremely short orifice (rather than a nozzle). Its Ст is 1.288 - again an extreme geometry not used in usual engineering applications. Despite these geometries being so extremely different, in Fig. 10 they are seen to agree with the second-order universal characteristic eq. (10). 


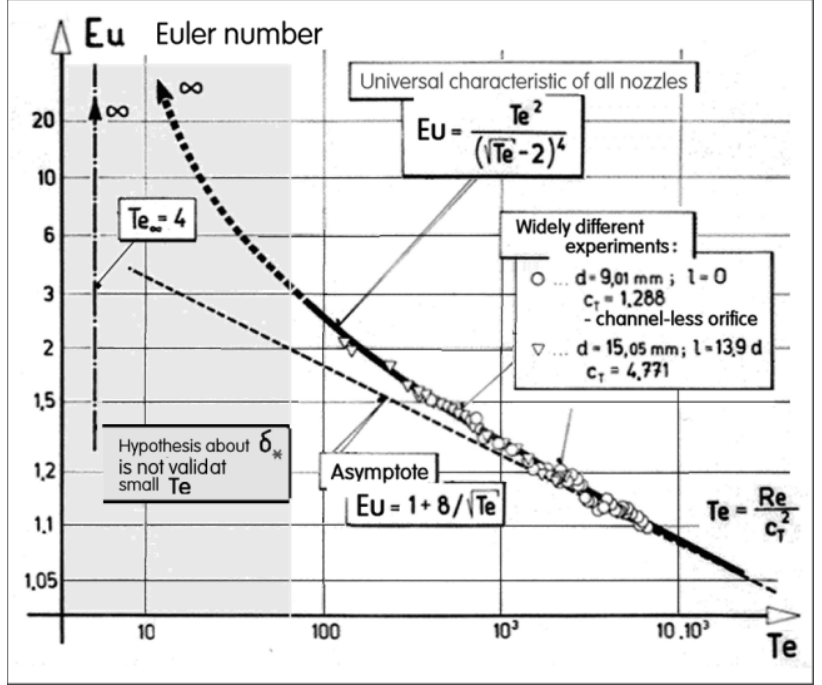

Fig. 10. Author's universal characteristic of all nozzles compared with two extreme cases, the shortest and the longest nozzle for which were by the author accumulated experimental pressure loss data.

\section{Application examples}

\subsection{Two nozzles in parallel, characterised by $Q$}

This is a rather simple case of a fluidic circuit solution problem. Classical approach is laborious step-by-step evaluation of velocities and pressures, usually complicated by initial absence of knowledge of the flow division into the two paths (note that the nozzles need not be identical or even of similar properties). Thus the computation has to progress in iteration cycles. Introduction of the dissipances $Q_{1}$ and $Q_{2}$ charac-terising the nozzles simplifies the procedure substantially. Presented at left in Fig. 11 is schematic representation of the two nozzles both connected to the same source of fluid - and hence applying at both nozzles the same constant specific energy drop $\Delta \mathrm{e}$. The solution steps in Fig. 11 shows the solution to be quite simple. It may be useful to introduce the concept of a unit consisting of the two nozzles and evaluate effective dissipance Qef of this unit. In the bottom left part of Fig. 11 is the expression for this unit's effective dissipance.
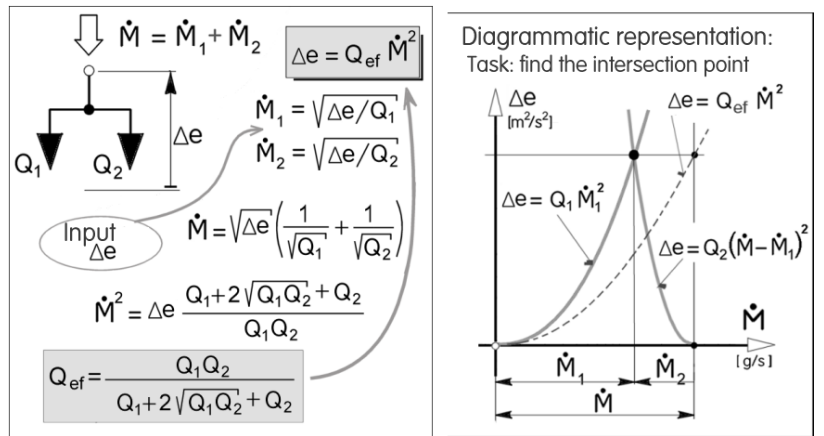

Fig. 11. An example of usefulness of nozzle characterisation by quadratic dissipances $\mathrm{Q}$. For a given specific energy drop $\Delta \mathrm{e}$ and dissipances $Q_{1}$ and $Q_{2}$ of the two nozzles the approach evaluates their mass flow rates. A useful step is to evaluate the effective dissipance $Q_{e f}$ of the unit consisting of the two nozzles.
It is a result of the sum of reciprocal square roots of the two dissipances. It is easy to evaluate the total mass flow rate through the unit as the sum of the two mass flow rates. The first step in the solution is evaluation of the two flows by inversions of eq. (5). Their sum is then squared - and this immediately leads to computing the value $\mathrm{Q}_{\text {ef. }}$ The procedure may be guided by the graphical solution shown in the right-hand side of Fig. 11. Two quadratic-parabola characteristics are drawn, with their origins on the horizontal mass flow rate axis - one of them is drawn on a transparent paper, which is then inverted and shifted along the horizontal axis until the intersection of the conditions (vertical positions of the interaction points) is fulfilled. Of course, real drawing the two parabolic curves and search for their intersection point is nowadays unlikely, but a mental picture of the two curves may serve as a useful support for the actual computation on a computer. The procedures of Fig. 11 may be easily generalised to the case of a larger number of parallel nozzles - note that coolant nozzles are often arranged in such clusters.

\subsection{Application example \#2: Nozzle characterised by dissipance $Q$ matched to its fluid supply source}

Solutions like the result in previous \#1 may assume a constant specific energy drop at the exit of fluid flow source. This may be done, but complicates the circuit by added pressure regulator - making the system less efficient, due to the loss in the source, and more expensive. Without regulator, the available specific energy (and of course also pressure in a simple incompressible case) decreases with increasing delivered flow rate. A realistic model of a fluid source with exit loading, shown in the bottom left part of Fig. 12, has in the output a fluidic resistor representative of the decrease. Its presence models the typical losses that the fluid must overcome on its way out to the load.

The task is to find the optimum delivery of fluid power from the supply to the nozzle - as shown schematically at left in Fig. 12. Behaviour of the nozzle is described by its dissipance $\mathrm{Q}_{\mathrm{N}}$, dependent upon the nozzle exit diameter $d$. If this diameter is small (the case of high nozzle dissipance) the delivered power is small because of the small nozzle exit flow rate. On the other hand, with a large-diameter, low dissipance nozzle the fluid flow issuing from the nozzle may be large, but the available pressure drop on it would be so low that the delivered power would be also uselessly small. Optimum behaviour of this simple circuit is obviously somewhere between these two extremes. It is defined by the maximum product of the two exit parameters, nozzle output mass flow rate and specific energy drop. The derivation in [17] is as follows:

The power $\dot{A}[\mathrm{~W}]$ delivered into the nozzle is

$$
\dot{\mathrm{A}}=\Delta \mathrm{e}_{\mathrm{Y}} \dot{\mathrm{M}}_{\mathrm{Y}}
$$

The characteristic of the nozzle is

$$
\Delta e_{Y}=a_{N} \dot{M}_{Y}^{2}
$$




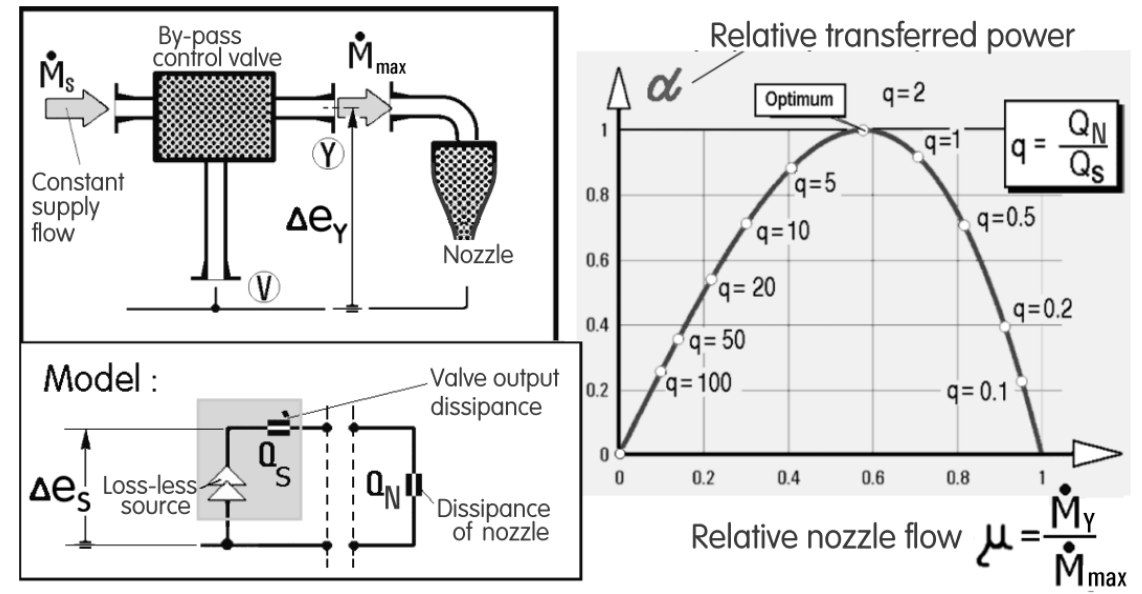

Fig. 12. An example of matching the nozzle, characterised by its dissipance $Q_{N}$, and its fluid supplying valve with output dissipance Qs. The ratio of the two dissipances $q$ is related in the unique manner to the ratio of supplied and by-passed flow rates, the dependence exhibiting an optimum for transferring maximum fluid power (product of mass flow rate and specific energy drop).

while the loading characteristic of the source is

$$
\Delta e_{Y}=\Delta e_{S}-a_{S} \dot{M}_{Y}^{2}
$$

(from the constant specific energy ${ }^{\Delta e_{S}}$ of an ideal lossless source is subtracted the internal loss inside the source). Combination of eqs. (12) and (13) is

$$
\mathrm{a}_{\mathrm{N}} \dot{\mathrm{M}}_{\mathrm{Y}}^{2}=\mathrm{a}_{\mathrm{S}} \dot{\mathrm{M}}_{\text {max }}^{2}-\mathrm{a}_{\mathrm{S}} \dot{\mathrm{M}}_{\mathrm{Y}}^{2}
$$

and the power delivered into the nozzle is

$$
\dot{\mathrm{A}}=\Delta \mathrm{e}_{\mathrm{S}} \dot{\mathrm{M}}_{\mathrm{Y}}-\mathrm{a}_{\mathrm{S}} \dot{\mathrm{M}}_{\mathrm{Y}}^{3}
$$

The optimum is defined by

$$
\frac{\mathrm{d} \dot{\mathrm{A}}}{\mathrm{d} \dot{\mathrm{M}}_{\mathrm{r}}}=0
$$

Applying this condition to eq. (15) results in

$$
\begin{gathered}
{ }^{\Delta e_{S}}=30_{S} \dot{m}_{Y}^{2} \\
\dot{m}_{Y}=\sqrt{\frac{\Delta e_{s}}{3 a_{S}}}
\end{gathered}
$$

It is useful to operate with relative, universally valid dimensionless vaslues.

$$
\mu=\frac{\dot{M}_{Y}}{\dot{M}_{\max }} \quad \alpha=\dot{A} / \dot{A}_{\max }
$$

- where theoretical maximum flow rate ideally obtainable from the source (at zero specific energy drop at the output terminal) is

$$
\dot{M}_{\text {max }}=\sqrt{\frac{\Delta \mathrm{e}_{\mathrm{s}}}{\mathrm{a}_{\mathrm{s}}}}
$$

- which, compared with eq. (18) means the optimum is at

$$
\mu_{\text {opt }}=\frac{1}{\sqrt{3}}
$$

while the optimum value of the loading factor $q-$ the ratio of nozzle and source dissipances is

$$
q=\frac{Q_{N}}{Q_{S}}
$$

in re-written eq.(14) is

$$
q \mu^{2}=1-\mu^{2}
$$

which means (Fig. 12)

$$
\mathrm{q}_{\mathrm{opt}}=2 \quad \mathrm{a}_{\mathrm{N}}=2 \mathrm{a}_{\mathrm{S}}
$$

\subsection{Application example \#3: Request for no spillover regime}

Difficulty with no-moving-part fluidic devices and circuits is their generally more laborious design than in of corresponding hydraulic and pneumatic devices with moving parts. In the latter cases the active mechanical component is placed to a position in which it dictates where the fluid must and must not go The undesirable other flowpaths are simply mechanically blocked.. In the pure, no-moving-part fluidics such blockage does not exist and the choice of desirable flow path among the existing ones depends on the proper distribution of pressure. At the same time, there are now increasingly often situations in which precise determination of the fluid flowpath is absolutely necessary. Typical for present-day fluidics and microfluidics are biomedical applications where the handled fluids are small samples of biological material - sometimes difficult to obtain (perhaps at a cost of pain to the patient) and at any rate the samples should be small. This means any spilling over of the sample into the wrong path - and even a small partial loss by this spillover, as suggested in Fig. 13 - is definitely unwelcome and sometimes forbidden. Another area of increasing popularity of fluidics is microchemistry. typical tasks there are discoveries of new substances (a case is discovery of new catalysts [3, 22]). For the reliability of the obtained results it is absolutely necessary absolutely necessary to prevent any crosscontamination between the very small handled amounts . 


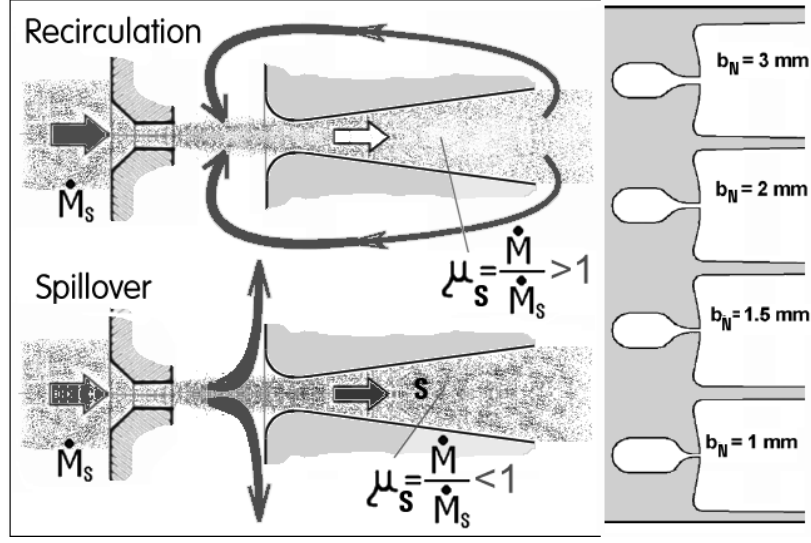

Fig. 13. In the absence of solid mechanical blockage in pure fluidics, it depends on the loading whether there will be jet pumping or spillover - or, mostly desired, neither of these two $=$ the no-spillover regime. The study in [17] used a family of nozzles (right) with equal depth and different exit widths.

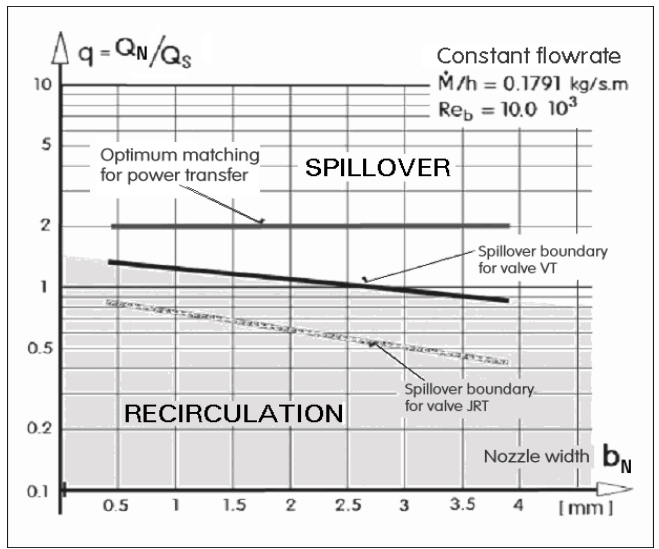

Fig. 14. Optimality criterion quite often imposed is request for the no-spillover regime. In this example from [17], various nozzles were connected to fluidic valve VT and JRT designs. Note that achieving the no-spillover $\mu \mathrm{s}=1$ was in both cases incompatible with the power transfer optimum $q=2$ of Fig. 12.

of the reactants. This is another typical task requiring precise adjustments of pressure distributions that can ensure proper resultant flow directions, either the recirculation or spilling the fluid over (Fig. 13) or, the most often demanded, the exact no-spillover regime. The circuit design had to develop relations for the characterisation quantities $\mathrm{Q}$ of the valve as well of the connected fluidic loads. As demonstrated on the example in Fig. 14 reproduced from [17], quite often the performance criteria are, unfortunately, mutually incompatible: For example, the no-spillover conditions, different for the two valves discussed in [17], cannot be achieved simultaneously with the power transfer optimum $\mathrm{q}_{\mathrm{opt}}=2$.

\subsection{Example \#4: Apparent $Q$ increase in pulsatile-flow}

Nonlinearity of nozzle characteristic causes phenomena that may surprise unprepared, especially those who intent to measure the time-mean pressure drop across a nozzle. If the flow contains, apart from the steady component, also a periodic pulsation component — such as the harmonic one in Fig. 15 - the measured time-mean pressure difference increases above the expected value. The nozzle apparently behaves as if its exit were becoming smaller: the pressure drop across it has increased. The effect is, in fact, a special case of flow rectification. In practice it is complicated by influence of capacitance of connected accumulation devices. The time-mean value of the pressure (or, in Fig. 16, the specific energy difference) increases because the secondpower component $\sin ^{2}(2 \pi \mathrm{ft})$ is everywhere

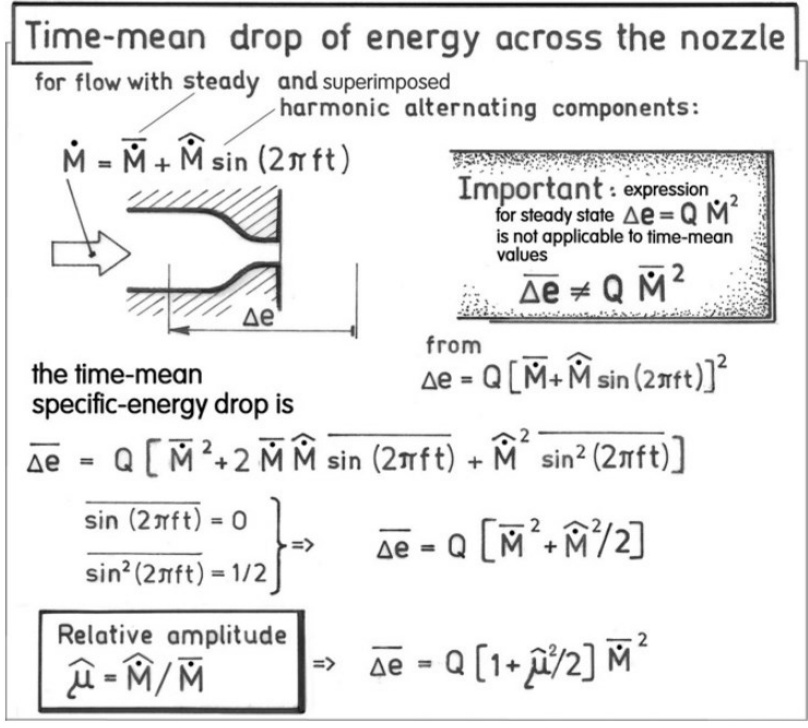

Fig. 15. Because of non-linearity of the nozzle characteristic, superposition of periodic pulsation (here the simple harmonic alternating flow) results in the nozzle behaving as if its timemean dissipance increases.

positive and adds an amount to the result - an amount proportional to the square of the relative amplitude of the alternating component.

Author performed simple experimental verifications of these results. A nozzle of exit diameter $d=6.03 \mathrm{~mm}$, with quadrant-shaped-shaped contraction corresponding to the nozzles presented in Fig. 7, but with zero channel length $l / \mathrm{d}=0$ - was connected to a positivedisplacement blower. This type of blowers supplies air at a constant adjusted flow rate in a flow rate not dependent on the loading connected to it. The other, pulsatory flow component was supplied by piston-type generator with the piston driven by the Scotch-yoke mechanism ensuring a simple harmonic flow oscillation. The time-mean pressure difference across the nozzle was measured by highly damped liquid micromanometer. Evaluated from the time-mean pressure drop was the effective dissipance Qef compared with the steady-state flow (no pulsation) nozzle dissipance $Q$. Each of these four experiments, the results of which are presented in Fig. 16, was run starting from the non-moving piston generator so that it was possible to check the initial value $Q$. Then the piston generator was switched on and the amplitude of piston motion was gradually increased. The stroke $s$ of the piston was kept constant $s / d=1.1610^{3}$. As expected on the basis of the derivation shown in Fig. 15, the time- 


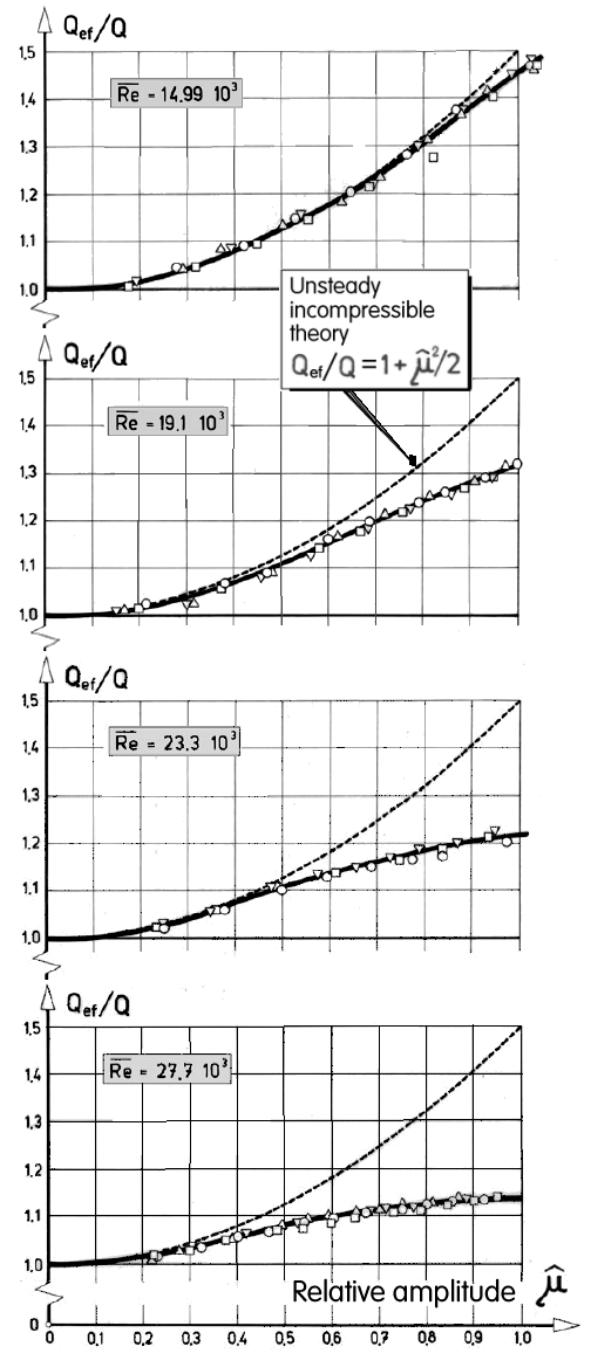

Fig. 16. Examples of time-mean pressure drops across the nozzle measured by the author. The values are smaller than the theory presented in Fig. 15. This pressure-decreasing effect was stronger with increased magnitude of the DC flow component (in the diagrams characterised by the time-mean Reynolds number - although later analysis has shown that this is not a Re effect).

mean pressure across the nozzle has gradually increased. Each of the four experiments finished when the maximum amplitude reached the magnitude of $A C$ component. Were the piston amplitude increased further, the air flow at some segment of the oscillation would reverse sign - obviously undesirable in this experiment because the used quadrant-shaped nozzle would exhibit different properties in the reverse flow direction. In the four experiments with results shown in Fig. 16 the agreement with the theory from Fig. 15 was satisfactory in the case with smallest flow rate. The time-man flow rate is in Fig. 16 characterised by the value of Reynolds number $\overline{\mathrm{Re}}$ - because the deviations from the theory were at that time thought to be a Reynolds-number effect (it should be emphasised that the disadvantage of the used first-order characterisation of the nozzle is the variation of $Q$ with varying $R e$ ).

The correct explanation of the pressure decreasing effect as seen in Fig. 16 was later provided in ref. [13]. It

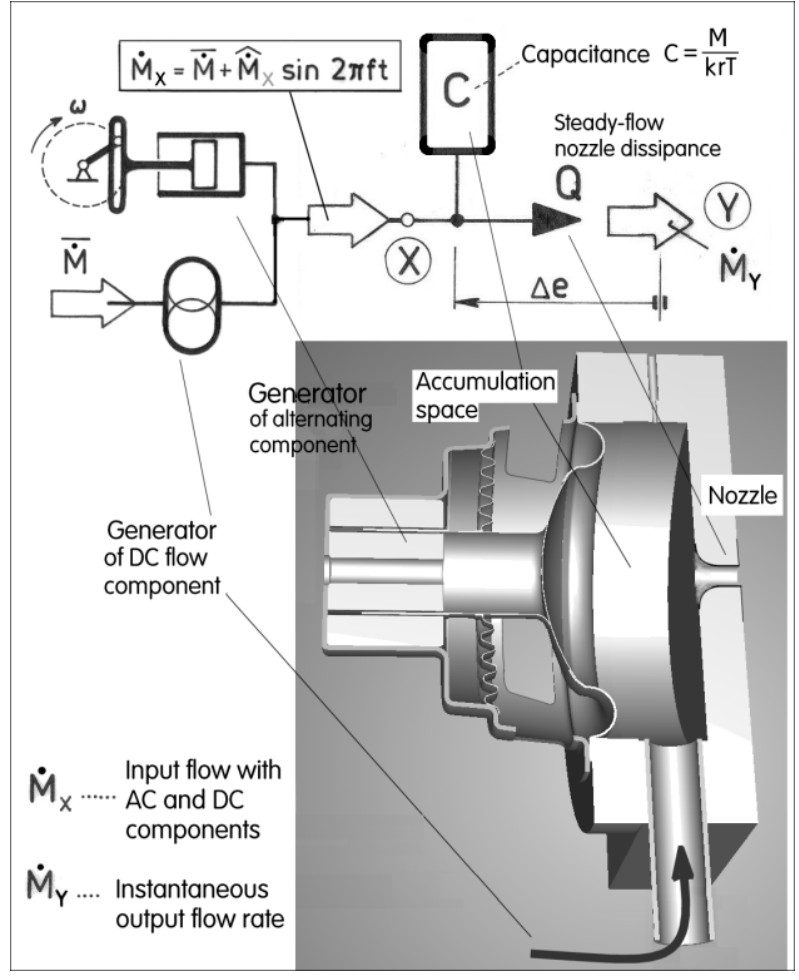

Fig. 17. An example and schematic representation of the test configurations used for investigating the effect of attached accumulation chamber. Instead of the original piston-type devices, with inertial-limited frequency range, these later devices used loudspeaker driver generating the AC flow component.

mulations space upstream from the nozzle for two purposes. One of them, is the usual flow recovery after the disturbance caused by the asymmetric inflow (note that in Fig. 17 the steady component comes through the tube from below). The other purpose is providing a space for the moving piston - or, in the present case the membrane of the speaker. The compression capability of this space is characterised by its capacitance C(Fig. 17) .

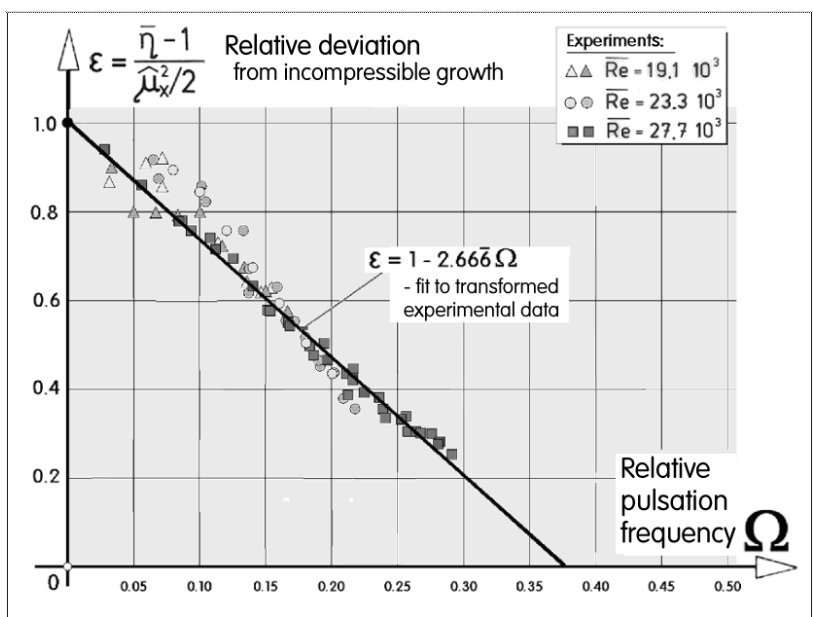

Fig. 18. Demonstration of the correctness of the explanation of the pressure decrease in Fig. 15 by the damping effect of the inevitable air-accommodating chamber $\mathrm{C}$ in Fig. 15. There is a universal linear law for the compressibility effect as a function of the relative pulsation frequency. 
It determines, together with the nozzle dissipance $\mathrm{Q}$, the characteristic time of the accumulation

$$
t_{c}=C Q \overline{M_{x}}
$$

- where $\overline{\bar{M}_{X}}$ is the constant time-mean input flow delivered by the DC flow generator.

The discussion in [13] has introduced several dimensionless variables by means of which it is possible to express the results. in general terms. One of them is the dimensionless frequency

$$
\Omega=\pi \mathrm{ft}_{\mathrm{c}}
$$

The diagram in Fig. 18 shows the universal dependence of the relative deviations from the incompressible expression (from Fig. 15) on this dimensionless frequency.

\section{Conclusions}

This paper surveys the parameters useful for characterising hydrodynamic properties of nozzles. There is actually a hierarchy of characterisation approaches. Known and so fat widely used is the simplest 0-th order characterisation eq. (9), which neglects frictional losses inside the nozzle. Later was introduced the $\mathbf{1}^{\text {st }}$ order characterisation by quadratic dissipance Q, which takes into account the internal (mainly friction) losses and considers them a constant. not Re-dependent eq. (8). Applicable within much wider range of Reynolds number values is the recently introduced $\mathbf{2}^{\text {nd }}$ order approach eq. $(1,9,10,12)$, which characterises a nozzle by its invariant coefficient $\mathrm{C}_{\mathrm{T}}$, in the expression eq. (10). Recently this second-order approach eq. (10) was extended for use in family of quasi-similar nozzles (in fact of dissimilar geometry) with properties described by secondary invariants, Fig. 7. Paper provides several examples of the practical applications nozzle computations using the characterisation.

\section{Acknowledgements}

Author obtained institutional support RVO:61388998 and also Czech Republic Grant Agency research grant Nr. 1708218S

\section{References}

1. V. Tesař, Application PO 17-85 for Certificate of Discovery, Czechoslovak Patent and Discovery Office, Prague April 1985

2. V. Tesař, chapter „Impinging Jets” pages. 191 - 231 in: Vortex Rings and Jets, Recent development in Near-Field Dynamics. Springer Science + Business Media, 2015

3. V. Tesař, Pressure-driven microfluidics, Artech House Publishers, Boston USA 2007

4. A. Hauptmann, I. Wagner, Metals and Mines, Studies in Archeology, British Museum Pub., London, 67 (2007)

5. Z. Váňa, Artia Praha 1983

6. B. C. Bobusch, Dissertation TU Berlin, 2015

7 H. Choi, W.-P. Jeon, J. Kim, Annular review of fluid mechanics, Vol. 49, 2008

8. W. B. J. Zimmerman, V. Tesař, European Patent. EP2081666, filed Oct. 2007

9. V.Tesař, Chemical Engineering Research and Design 861253 (2008)

10. V. Tesař, Chemical Engineering Research and Design 881433 (2010)

11. V. Tesař, Sensors and Actuators A, 263, 147 (2017)

12. V. Tesař, Sensors and Actuators A, 248246 (2016)

13. V. Tesař, Sensors and Actuators A, 179211 (2012)

14. V. Tesař, J. Kordík, Sensors and Actuators A, 149 255 (2009)

15. V. Tesař, J. Kordík, Sensors and Actuators A, 199 379 (2013)

16. V. Tesař, J. Kordík, Sensors and Actuators A, 199 391 (2013)

17. V. Tesař, Chemical Engineering Research and Design, 87817 (2009)

18. E. Jarkovský, Foundations of practical computation of orifices, nozzles, and Venturi tubes, SNTL Prague (1958)

19. V. Tesař, Zeitschrift für Angewandte Mathematik und Mechanik, 77, 333 (1997)

20. H. Blasius, Grenzschichen in Flussigkeiten mit kleiner Reibung, Teubner 1907,

21. V. Tesař, Chapter in Microfluidics: History, Theory, and Applications, Springer-Verlag, Wien-New York 2006

22. V. Tesar̆, Role of microfluidics in discovering new marketable substances - a survey, Proc. of 19th Internat. Conf., p. 614, Svratka, Czech Republic, May 2013

23. V. Tesař, J. Kordík, Sensors and Actuators A, 191 34 (2013) 